\title{
STOCHASTIC STABILITY AND THE SPIN GLASS PHASE. THE STATE OF ART FOR MEAN FIELD AND FINITE DIMENSIONAL MODELS
}

\author{
P. CONTUCCI \\ Department of Mathematics, Alma Mater Studiorum \\ University of Bologna, Italy \\ E-mail: pierluigi.contucci@unibo.it \\ http://www.dm.unibo.it/ contucci
}

\begin{abstract}
Some invariances under perturbations of the spin glass phase are introduced, their proofs outlined and their consequences illustrated as factorization rules for the overlap distribution. A comparison between the state of the art for mean field and finite dimensional models is shortly discussed.

Keywords: Disordered systems, Spin Glasses, Structural Identities, Ultrametricity
\end{abstract}

Factorisation laws for observable quantities are very important both from theoretical and experimental perspective in statistical mechanics and quantum field theory. Their rigorous proof is then a crucial step toward the understanding of a theory and its specific models. Examples are the Wick law in Euclidian quantum field theory, the multiplicative factorisation of the Curie Weiss theory of ferromagnetism, and more recently, the Parisi theory of the spin glass phase. This last case presents a factorisation structure that has been one of the most intriguing features of theoretical physics in the last decades and has found applications in many areas as diverse as computer sciences, biology and economics by means of new ideas and methods in probability, machine learning, optimisation etc.

In this short note we give a brief account of its nature and its origins introducing, via a mathematically elementary method based on sound physical ideas, both its form and a guide to its proof.

We consider configurations of $N$ Ising spins

$$
\sigma=\left\{\sigma_{i}\right\}, \quad \tau=\left\{\tau_{i}\right\}, \quad \ldots
$$

and introduce a centered Gaussian Hamiltonian $H_{N}(\sigma)$ defined by the covariance

$$
\operatorname{Av}\left(H_{N}(\sigma) H_{N}(\tau)\right)=N c_{N}(\sigma, \tau) .
$$


For all $\beta>0$ we are interested in the random probability measure

$$
p_{N}(\sigma)=\frac{e^{-\beta H_{N}(\sigma)}}{\sum_{\sigma} e^{-\beta H_{N}(\sigma)}}
$$

Important examples are:

the Sherrington-Kirkpatrick model

$$
c_{N}(\sigma, \tau)=\left(\frac{1}{N} \sum_{i=1}^{N} \sigma_{i} \tau_{i}\right)^{2}
$$

and the Edwards-Anderson model

$$
c_{N}(\sigma, \tau)=\frac{1}{d N} \sum_{|i-j|=1} \sigma_{i} \sigma_{j} \tau_{i} \tau_{j}
$$

Quantities of interest include:

- the pressure

$$
P_{N}(\beta)=\operatorname{Av} \log \sum_{\sigma} e^{-\beta H_{N}(\sigma)}
$$

- the moments

$$
\begin{gathered}
\operatorname{Av}\left(\frac{\sum_{\sigma, \tau} c_{N}(\sigma, \tau) e^{-\beta\left[H_{N}(\sigma)+H_{N}(\tau)\right]}}{\sum_{\sigma, \tau} e^{-\beta\left[H_{N}(\sigma)+H_{N}(\tau)\right]}}\right)= \\
=<c>_{N}:=\int c p_{N}(c) d c \\
\operatorname{Av}\left(\frac{\sum_{\sigma, \tau, \gamma} c_{N}(\sigma, \tau) c_{N}(\tau, \gamma) e^{-\beta\left[H_{N}(\sigma)+H_{N}(\tau)+H_{N}(\gamma)\right]}}{\sum_{\sigma, \tau, \gamma} e^{-\beta\left[H_{N}(\sigma)+H_{N}(\tau)+H_{N}(\gamma)\right]}}\right)=<c_{12} c_{23}>_{N}= \\
:=\int c_{12} c_{23} p_{N}^{(12),(23)}\left(c_{12}, c_{23}\right),
\end{gathered}
$$

etc. and, especially, the

- joint overlap distribution

$$
p_{N}^{(12),(23), \ldots,(k l), \ldots}\left(c_{12}, c_{23}, \ldots, c_{k l}, \ldots\right) .
$$

The Parisi theory for spin glasses (also known as Replica Symmetry Breaking theory), is based on the following "factorization" assumptions (see [1]) in the thermodynamic limit:

- Replica Equivalence, e.g.:

$$
\begin{aligned}
& p^{(12),(23)}\left(c_{12}, c_{23}\right)=\frac{1}{2} p\left(c_{12}\right) \delta\left(c_{12}-c_{23}\right)+\frac{1}{2} p\left(c_{12}\right) p\left(c_{23}\right) \\
& p^{(12),(34)}\left(c_{12}, c_{34}\right)=\frac{1}{3} p\left(c_{12}\right) \delta\left(c_{12}-c_{34}\right)+\frac{2}{3} p\left(c_{12}\right) p\left(c_{34}\right)
\end{aligned}
$$


- Ultrametricity, e.g.:

$$
\begin{gathered}
p^{(12),(23),(31)}\left(c_{12}, c_{23}, c_{31}\right)= \\
\delta\left(c_{12}-c_{23}\right) \delta\left(c_{23}-c_{31}\right) p\left(c_{12}\right) \int_{0}^{c_{12}} d c p(c) \\
+\theta\left(c_{12}-c_{23}\right) \delta\left(c_{23}-c_{31}\right) p\left(c_{12}\right) p\left(c_{23}\right) \\
+2 \text { cyclic permutations }
\end{gathered}
$$

Replica Equivalence and Ultrametricity allows to reconstruct the $p^{(\{k l\})}\left(\left\{c_{k l}\right\}\right)$ starting from $p(q)$. This clearly means that we have a complete factorization and we are in presence of a typical mean field picture. Similar examples were the validity of the Wick rule for fields expectation in Euclidean quantum field theory, or the Boltzmann-Gibbs complete factorization for the Curie Weiss model.

We summarise here the results (see [2]) on a rigorous version of the linear response theory applied to the spin glass phase. The stability method in Statistical Mechanics works by identifying a small (yet non-trivial) deformation of the system, prove that in the large volume limit the perturbation vanishes and, by means of the linear response theory, compute the relations among observable quantities. This method leads to interesting consequences and applications because it reduce the apriori degrees of freedom of a theory. For instance it shows that ferromagnetic mean field models have a magnetisation which is a full order parameter for the theory.

More specifically in classical models one starts, for smooth bounded functions $f$ of spin configurations, from the counting measure

$$
\mu_{N}(f)=\frac{1}{2^{N}} \sum_{\sigma} f(\sigma)
$$

and defines the equilibrium state

$$
\omega_{\beta, N}(f)=\frac{\mu_{N}\left(f e^{-\beta H_{N}}\right)}{\mu_{N}\left(e^{-\beta H_{N}}\right)}
$$

By considering the Hamiltonian per particle

$$
h_{N}(\sigma)=\frac{H_{N}(\sigma)}{N}
$$

the classical perturbed state is defined by

$$
\omega_{\beta, N}^{(\lambda)}(f)=\frac{\omega_{\beta, N}\left(f e^{-\lambda h_{N}}\right)}{\omega_{\beta, N}\left(e^{-\lambda h_{N}}\right)} .
$$

Since the perturbation amounts to a small change in the temperature

$$
\omega_{\beta, N}^{(\lambda)}(f)=\omega_{\beta+\frac{\lambda}{N}, N}(f)
$$


one has that, a part for isolated singularity points, in the thermodynamic limit

$$
\frac{d \omega_{\beta, N}^{(\lambda)}(f)}{d \lambda} \rightarrow 0 .
$$

One may easily show that the previous property implies that for the Curie-Weiss model in zero magnetic field

$$
\omega_{\beta}\left(\sigma_{1} \sigma_{2} \sigma_{3} \sigma_{4}\right)=\omega_{\beta}\left(\sigma_{1} \sigma_{2}\right)^{2} .
$$

The previous approach lead to the concept of Stochastic Stability when applied, suitably adapted, to the spin glass phase. Consider, for smooth bounded function $f$ of $n$ spin configurations, the quenched equilibrium state

$$
<f>_{\beta, N}=\operatorname{Av}\left(\frac{\sum_{\sigma} f(\sigma) e^{-\beta H_{N}}}{\sum_{\sigma} e^{-\beta H_{N}}}\right)
$$

define the deformation as:

$$
<f>_{\beta, N}^{(\lambda)}=\frac{<f e^{-\lambda h_{N}}>}{<e^{-\lambda h_{N}}>} .
$$

We observe that the previous deformation is not a simple temperature shift. In fact:

$$
<f>_{\beta, N}^{(\lambda)}=\frac{\operatorname{Av}\left(\frac{\sum_{\sigma} f(\sigma) e^{-(\beta+\lambda / N) H_{N}}}{\sum_{\sigma} e^{-\beta H_{N}}}\right)}{\operatorname{Av}\left(\frac{\sum_{\sigma} e^{-(\beta+\lambda / N) H_{N}}}{\sum_{\sigma} e^{-\beta H_{N}}}\right)},
$$

nevertheless the system is still stable under it. We can state our main result as follows. The spin glass quenched equilibrium state is stable with respect to the deformation defined above in the sense that, a part for isolated singularity points, in the thermodynamic limit

$$
\frac{d}{d \lambda}<f>_{\beta, N}^{(\lambda)} \rightarrow 0
$$

moreover the previous stability property implies (by use of the integration by parts techinque) that the following set of identities (Ghirlanda-Guerra) holds:

$$
\begin{gathered}
<f c_{1, n+1}>_{\beta, N}=\frac{1}{n}<f>_{\beta, N}<c>_{\beta, N}+ \\
+\frac{1}{n} \sum_{j=2}^{n}<f c_{1, j}>_{\beta, N},
\end{gathered}
$$

where the term $c_{1, n+1}$ is the overlap between a spin configuration of the set $\{1,2, \ldots, n\}$ and and external one that we enumerate as $(n+1)$-nth, and $c_{1, j}$ is the overlap between two generic spin configurations among the $n$ 's.

The proof ideas can be easily summarized by the study of three quantities and their differences which encode the fluctuation properties of the spin glass system:

$$
\operatorname{Av}\left[\omega\left(H_{N}^{2}\right)\right],
$$




$$
\begin{aligned}
& \operatorname{Av}\left[\omega\left(H_{N}\right)^{2}\right], \\
& \operatorname{Av}\left[\omega\left(H_{N}\right)\right]^{2} .
\end{aligned}
$$

The result is obtained by two bounds:

- bound on averaged thermal fluctuations

$$
\operatorname{Av}\left[\omega\left(H_{N}^{2}\right)-\omega\left(H_{N}\right)^{2}\right] \leq c_{1} N
$$

obtained by stochastic Stochastic Stability method (see [3]) by showing that the addition of an independent term of order one to the Hamiltonian is equivalent to a small change in temperature of the entire system:

$$
\begin{gathered}
\beta H_{N}(\sigma) \rightarrow \beta H_{N}(\sigma)+\sqrt{\frac{\lambda}{N}} \tilde{H}_{N}(\sigma) \\
\beta \rightarrow \sqrt{\beta^{2}+\frac{\lambda}{N}}
\end{gathered}
$$

- bound on disorder fluctuations

$$
\begin{gathered}
U=\omega\left(H_{N}\right) \\
\operatorname{Av}\left(U^{2}\right)-\operatorname{Av}(U)^{2} \leq c_{2} N,
\end{gathered}
$$

which is the self averaging of internal energy and can be proved from self averaging of the free energy (with martingale methods or concentration of measures).

A recent achievement (see [4]) shows that the validity of the previous identities extended to the moments of all orders, i.e. their validity in distribution, is compatible only with an ultrametric distribution of the overlaps. Since the validity of the distributional identities was previously shown by Talagrand for the mean field models, Panchenko's result proves the ultrametricity result for those models.

The next challenge is to work with models in finite dimensions (especially $d=3$ ) who are more directly related to physics. For those models a proof of the distributional factorisation laws is in progress [5]. One has to stress nevertheless that its achievement wouldn't end the discussion about the triviality issue of the EdwardsAnderson model because it leaves still open the nature of the single overlap distribution. The rigorous proof for its non-triviality, available so far, works only for the general mean field case.

Acknowledgments. This work was presented at the ICMP12 in Aalborg. It is a pleasure to thank the organisers for both the invitation and the opportunity to give the talk and to write this contribution. 


\section{References}

[1] M.Mezard, G.Parisi and M.A.Virasoro, Spin Glass Theory and Beyond (World Scientific, Singapore, 1987)

[2] P.Contucci, C. Giardina and C. Giberti, Eur. Lett., 96, 17003, (2011).

[3] M.Aizenman and P.Contucci Jou. of Stat. Phys., 92, 765, (1998).

[4] D.Panchenko Ann. of Math., 177, 383 (2013)

[5] P.Contucci, E.Mingione and S.Starr. Work in proggress. 